NASA Technical Memorandum 105180

AIAA-91-2459

\title{
Experimental Study of Cross-Stream Mixing in a Cylindrical Duct
}

\author{
A. Vranos, D.S. Liscinsky, and B. True \\ United Technologies Research Center \\ East Hartford, Connecticut
}

and

J.D. Holdeman

Lewis Research Center

Cleveland, Ohio

Prepared for the

27th Joint Propulsion Conference

cosponsored by the AIAA, SAE, ASME, and ASEE

Sacramento, California, June 24-27, 1991 


\title{
EXPERIMENTAL STUDY OF CROSS-STREAM MIXING \\ IN A \\ CYLINDRICAL DUCT
A. Vranos, ${ }^{*}$ D.S. Liscinsky, ${ }^{\star \star}$ and B. True ${ }^{* * *}$
United Technologies Research Center
East Hartford, CT 06108

\author{
J.D. Holdemant \\ NASA Lewis Research Center \\ Cleveland, $\mathrm{OH} \quad 44135$
}

\begin{abstract}
An experimental investigation of cross stream injection and mixing has been conducted with application to a low $\mathrm{NO}_{x}$ combustor for the HSCT. Mixing in a cylindrical chamber has been studied for transverse injection from slanted slot and round orifice injectors. Momentum ratio, density ratio, and injector geometry were the primary variables. Slanted slots of various size, aspect ratio, and number were studied. Quantitative measurement of injectant concentration distributions were obtained by planar digital imaging of the Miescattered light from an aerosol seed uniformly mixed with the injectant. The unmixedness, defined as the ratio of the r.m.s. concentration fluctuation to mean concentration in a plane perpendicular to the main flow direction, was found to be primarily a function of momentum ratio and injector spacing. An optimum spacing is indicated. Unmixedness is also a function of orifice size, or mass flow ratio, but the mass flow dependence can be accounted for by normalizing the unmixedness with its maximum theoretical value. The data indicate that a density ratio greater than unity retards mixing. It was found that above a certain momentum flux ratio, mixing with slanted slot injectors was better than with round hole injectors.
\end{abstract}

Video tapes of the mixing at very low stream velocity indicate different jet/jet and jet/mainstream interactions for the two types of injectors.

\section{Nemenclature}

\begin{tabular}{|c|c|}
\hline$A_{j}$ & orifice area \\
\hline$\underline{C}_{d}^{\prime}$ & orifice discharge coefficient \\
\hline C & $\begin{array}{l}\text { fully mixed mass fraction } \\
\begin{array}{l}\left(=\left(w_{j} / w_{m}\right) /\left(1+w_{j} / w_{m}\right)\right. \\
\left.=\theta_{E B}, \text { Ref. } 1-5\right)\end{array}\end{array}$ \\
\hline D & mixing section diameter \\
\hline $\mathbf{F}$ & $\begin{array}{l}\text { segregation parameter } \\
\quad=\sqrt{ }[(1-\bar{C}) / \bar{C}] ; \text { eq. } 3\end{array}$ \\
\hline$J$ & $\begin{array}{l}\text { jet-to-mainstream momentum } \\
\text { flux ratio }=\rho_{\mathrm{j}} \mathrm{V}_{\mathrm{j}}^{2} / \rho \mathrm{m} \mathrm{V}_{\mathrm{m}}{ }^{2}\end{array}$ \\
\hline$w_{j} / w_{m}$ & $\begin{array}{l}\text { jet-to-mainstream mass flow } \\
\text { ratio }\end{array}$ \\
\hline$S$ & $\begin{array}{l}\text { spacing between orifice mid- } \\
\text { points }\end{array}$ \\
\hline$V_{m}$ & mainstream velocity \\
\hline $\begin{array}{l}V_{j} \\
X / D\end{array}$ & jet velocity $=m_{j} / \rho_{j} A_{j} C_{d}$ \\
\hline$x / 0$ & ( $X=0$ at mid-point of orifice) \\
\hline & $=\left(\mathrm{C}_{\mathrm{rms}} / \mathrm{C}_{\text {avg }}\right)^{/ \mathrm{F}}$ \\
\hline
\end{tabular}

* Consulting Scientist, Member AIAA

** Research Scientist, Member AIAA

*** Senior Laboratory Technician

$\dagger \quad$ Senior Research Engineer, Member AIAA 


\section{Introduction}

An experimental investigation of crossstream jet injection and mixing has been conducted with application to a low $\mathrm{NO}_{x}$ combustor for HSCT. The configurations tested were in accordance with an RQL (rich burn, quick mix, lean burn) concept. Primary concern was the evaluation of the separate effects of injector geometry and jet flow parameters such as momentum and density ratio on mixedness in non-reacting flow. Slot and round hole radial inflow injectors, as currently envisioned for the quick mix section of the combustor, were investigated.

The work reported herein was concerned with mixing in a duct of circular cross-section, akin to a can type combustor, in support of current, low $\mathrm{NO}_{x}$ combustor development programs at NASA LeRC and UTRC. A current phase of the mixing studies is concerned with mixing in a duct of rectangular cross section. The objective of the current phase is to develop rapid mixing configurations which can be incorporated into a subsequent annular combustor development program under the NASA Aero-Propulsion Technology Research Program.

Cross flow mixing has been studied by many investigators for a variety of flow configurations and applications. An extensive review of past work is not intended here. However, the application and objectives are most closely related to the investigations reported in Holdeman et al. ${ }^{1-5}$. These studies identified the effects of momentum flux ratio, injector shape, spacing, and location and duct shape on mixing in a rectangular sector geometry with application to gas turbine combustor exit temperature control. The objective of the current investigation is to develop rapid mixing techniques essential for $\mathrm{NO}_{x}$ control in an RQL configuration. Planar imaging is used to screen a large number of flow configurations and to extend the variables investigated by Holdeman et al. In particular, the present investigation is concerned with higher mass flow ratios typical of an HSCT combustor

\section{Experimental}

\section{Apparatus}

Figure 1 is a schematic of the apparatus with the baseline (wasp-waist) mixing section installed. The apparatus consists of three sections: (1) an inlet pipe, (2) a mixing section, and (3) a measuring section with diameters in the ratio of 6:5:7, respectively, as shown in Fig. 1.

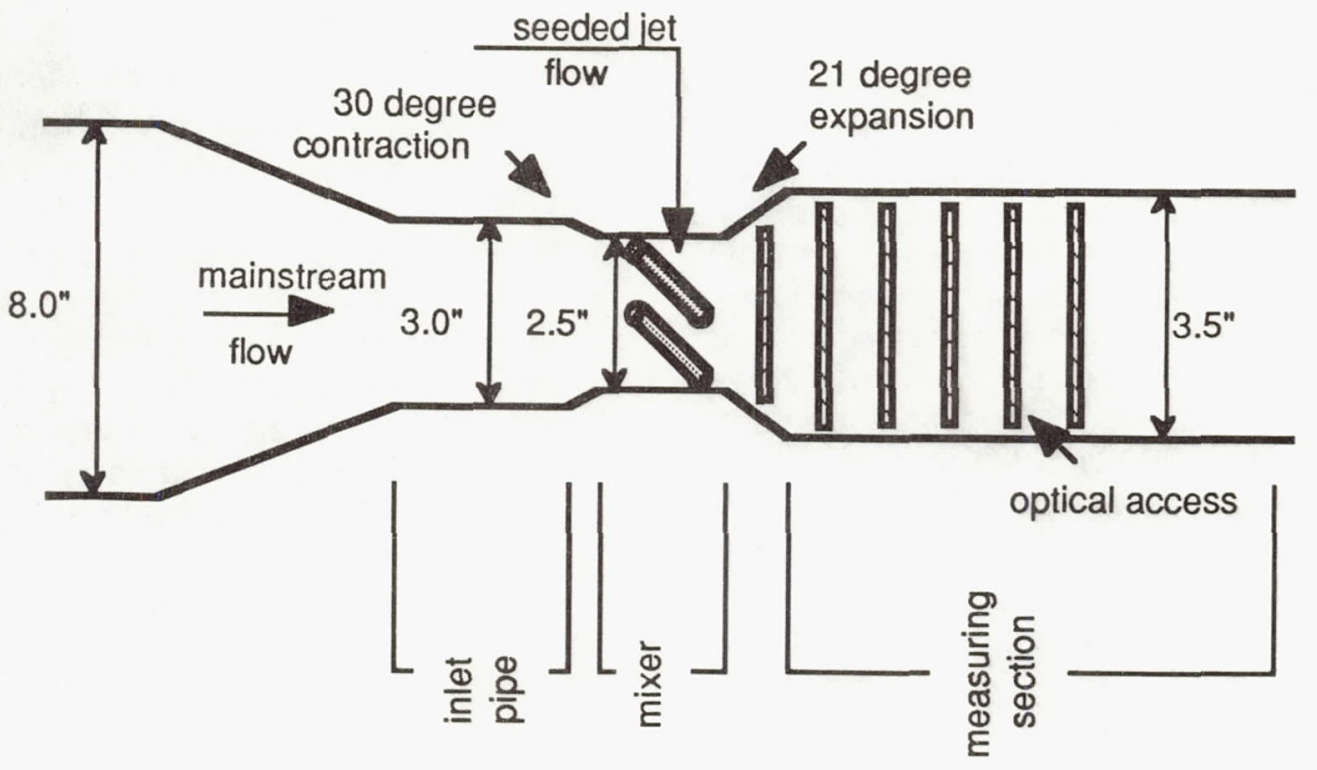

Figure 1: RQL Test Apparatus 
The mainstream flow is conditioned by a series of perforated plates and screens followed by a $7: 1$ contraction ratio nozzle. The flow velocity is uniform within $\pm 2 \%$ at the nozzle exit and was held constant at $10 \mathrm{ft} / \mathrm{sec}$ in all cases. The nozzle is attached to a 3 inch i.d. inlet pipe 6 inches in length. Turbulence intensity was not measured. The inlet pipe attached directly to the mixer. Air to the mixer is plenum fed. The two flows were metered with separate venturi meters supplied by 400 psi air. The density of either stream was modified by adding helium through a third venturi well upstream of the test section. The exhaust section downstream of the mixer contains a series of thin (1/16 inch wide) slits which allow optical access at one inch intervals. The first slit is 1.5 inches from the centerline of the mixing section $(X / D=0.6$ where $D$ is the diameter of the mixing section).

\section{Mixing Sections}

Various mixing sections were assembled using interchangeable parts. The four mixer configurations tested are shown in Table 2. The diagram indicates the relative sizes of the mixer orifices. Each mixer had 2 to 12 equally spaced orifices located along the circumference in the mid-plane of the mixer. As many of these orifices as desired could be rendered inactive by blanking, so the symmetric configurations available had $2,3,4,6$, or 12 orifices. The inside diameter and length of each mixer was 2.5 inches. The number of orifices was changed by blocking some orifices with tape. The slots had straight sides with circular ends.

Previous work 6,7 has indicated that desirable mixing section configurations contract between the rich and lean zones in order to minimize $\mathrm{NO}_{x}$. Therefore, two different geometries were tested: (1) a waspwaist configuration, in accordance with previous experience, and (2) a constant area configuration, in order to eliminate duct geometry as a variable. In the wasp-waist configuration, shown in Fig. 1, the mainstream contracts through a $30 \mathrm{deg}$ angle upstream of the mixer, and expands at $21 \mathrm{deg}$ to a 3 inch duct downstream of the mixer. The straight configuration maintains a 2.5 inch diameter before and after the mixer.

\section{Data Acquisition}

Planar digital imaging was used to measure mixing rates. Concentration distributions were measured in a plane normal to the duct axis at several downstream stations for each test configuration. The light scattering technique can be summarized as follows: The jet flow is marked with an oil aerosol ( $\mu \mathrm{m}$ size particles). A thin light sheet $(0.5 \mathrm{~mm}$ thick) is created using a $2 \mathrm{~W}$ argon-ion laser and a rotating mirror. The flowfield is illuminated by directing the light sheet through one of the imaging ports. A camera, located in the flow downstream of the mixer (end-on view), is

\begin{tabular}{|c||c||c||c||c|}
\hline Mixer \# & $\mathbf{1}$ & $\mathbf{2}$ & $\mathbf{3}$ & $\mathbf{4}$ \\
\hline Geometry & $45 \mathrm{deg}$ slot & $45 \mathrm{deg}$ slot & $45 \mathrm{deg}$ slot & round hole \\
\hline Length (in) & 1.250 & 0.620 & 1.250 & 0.342 \\
\hline Width (in) & 0.312 & 0.156 & 0.156 & 0.342 \\
\hline Area (sq in) & 0.369 & 0.092 & 0.190 & 0.092 \\
\hline Aspect ratio & $4: 1$ & $4: 1$ & $8: 1$ & $1: 1$ \\
\hline
\end{tabular}

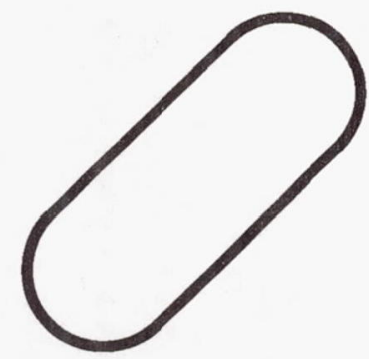

slot type 1

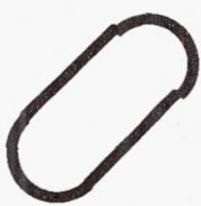

slot type 2

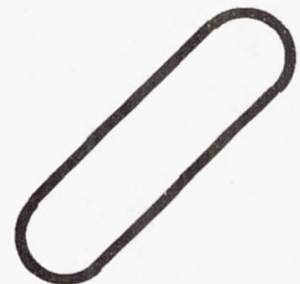

slot type 3

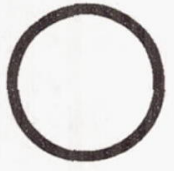

hole

Table 2: Mixer Configurations 
programmed to make exposures coincident with the sweep of the beam thru the flow field. The image is then digitized and sent to a computer for storage. The digitized light intensity is proportional to the number of particles in the measurement volume. When one of two streams is marked, the light intensity of the undiluted marked fluid represents mole fraction unity. For a more detailed discussion of the technique see Ref. 8.

Three different cameras were used for planar imaging. All three cameras gave essentially equivalent results, despite differences in operating characteristics. The camera used for the bulk of the data acquisition was a thermoelectrically cooled CCD (CC200, Photometrics, Ltd.). Spatial resolution was $0.01 \times 0.01 \times 0.02 \mathrm{inch} / \mathrm{pixel}$ in a data frame containing 122,500 pixels (350 x 350 format). Each image represents a high resolution time-averaged mass fraction distribution for a 5-10 second exposure. Comparison of results on the basis of mean mass fraction distributions allowed relative evaluation of a large number of flow configurations. Instantaneous concentration distributions were obtained using a a low light level vidicon camera. Exposure time was $10 \mu \mathrm{s}$. A typical example of the time-resolved data is shown in Fig. 2. All of the data were recorded and processed on a Macintosh II computer.

If undiluted jet fluid is present in the section sampled, then the measured distributions are absolute mole fractions. This is generally not the case, so measured distributions are relative. If absolute mole fractions are needed, the true mole fraction must be known at at least one point in the measurement plane.

Since unmixedness, as defined below, depends upon relative light intensities, it was possible to compare various configurations without knowledge of absolute mole fraction. However, in some cases the digital image (light signal) was converted to mole fraction by calibration. A trace amount of methane was introduced into the jet flow as a marker. Methane concentration, measured with an online hydrocarbon analyzer, provided absolute

Figure 2: Typical Instantaneous Mass Fraction Distributions

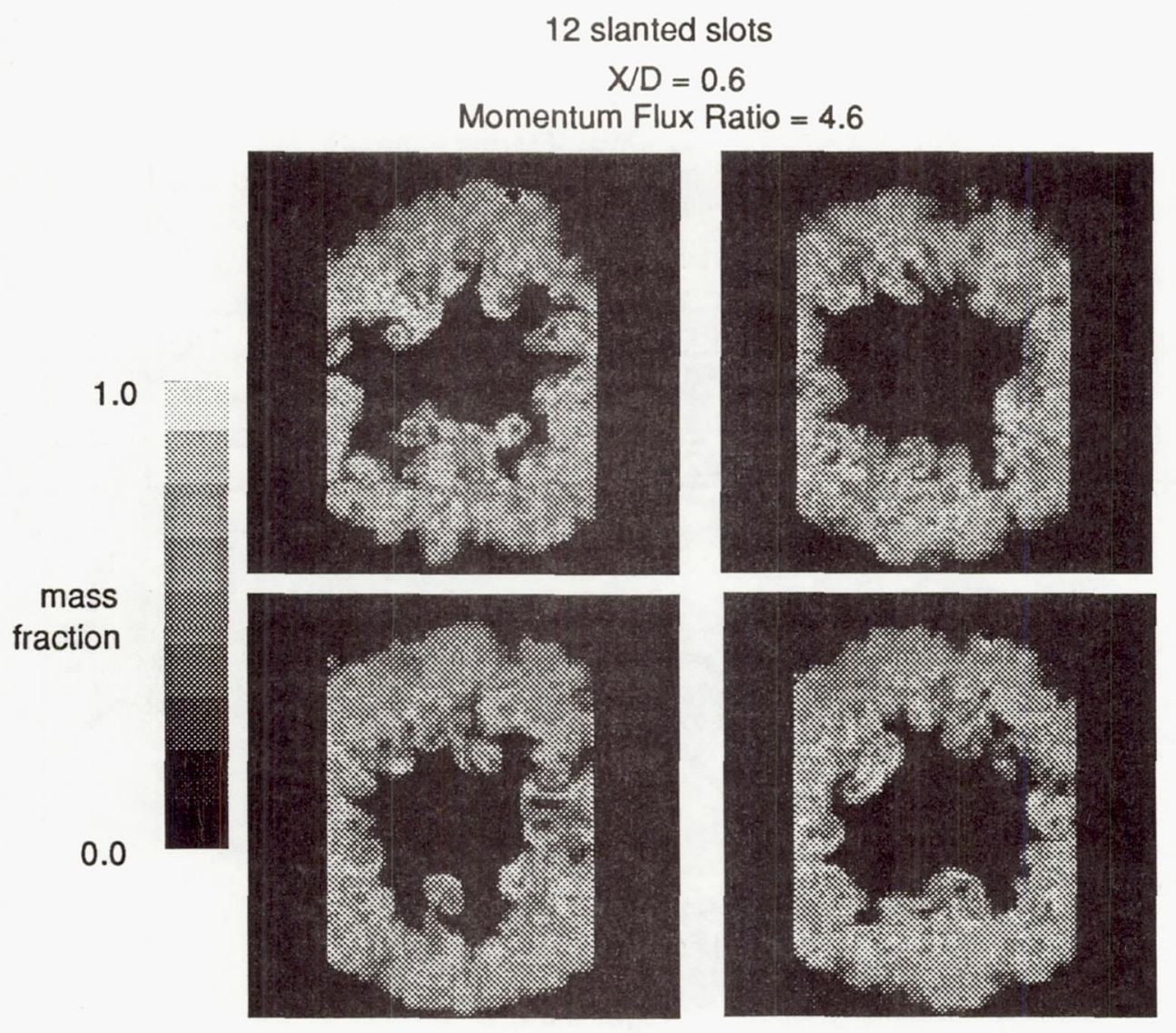


mole fraction at the sampling location. This measurement allowed conversion of the scattered light intensity measurement to absolute mole fraction (which can then be converted to mass fraction, if desired). For those frames where gas sampling was not available, the calibration was achieved by assuming that the average intensity across the duct was directly proportional to the fully mixed mole fraction.

\section{Data Reduction}

The purpose of this investigation was to screen rapidly the mixing efficiency of a variety of flow and geometric configurations. Mixing efficiency was measured by a parameter, $\mathbf{X}$, a macroscopic measure of the mixedness or degree of homogeneity of the gas. The mixing parameter $\mathbf{X}$ is well suited for making comparisons of systems with different fully mixed concentrations or the same fully mixed concentration but different initial/boundary conditions.

The unmixedness is defined as:

$$
\begin{aligned}
& \frac{\dot{c}}{\overline{\bar{c}}}=\frac{\sqrt{\frac{1}{n} \frac{1}{m} \sum_{j=1}^{n} \sum_{i=1}^{m}\left(C_{i j}-\overline{\bar{C}}\right)^{2}}}{\overline{\bar{C}}} \\
& \text { where, } \\
& n=\text { number of images in data set } \\
& \mathrm{m}=\text { number of pixels in each image } \\
& \overline{\bar{C}}=\frac{1}{n} \frac{1}{m} \sum_{j=1}^{n} \sum_{i=1}^{m} C_{i j} \\
& =\text { average over all points } \\
& \text { (all pixels, all realizations) } \\
& \mathrm{C}^{\prime}=\text { rms fluctuation }
\end{aligned}
$$

The relative unmixedness, $\mathbf{X}$, is defined as:

$$
X=\left(C^{\prime} / \overline{\bar{C}}\right) / F
$$

where,

$$
\begin{aligned}
\mathbf{F} & =\sqrt{\frac{1-\bar{C}}{\bar{C}}}=\left(w_{j} / w_{m}\right)^{-1 / 2} \\
\bar{C} & =\text { fully mixed mass fraction } \\
& =\text { fraction of total flow that enters } \\
& \text { through the jets }
\end{aligned}
$$

In Eq. (1), ( $\left.C_{i j}-\overline{\bar{C}}\right)$ is the difference between the instantaneous mass fraction at a particular $x, y$ location in a measuring plane perpendicular to the duct axis and the mean value in that plane, $\overline{\bar{C}}$. Summation is with respect to all realizations of the flow at all measuring locations in the plane. The segregation parameter $\boldsymbol{F}$ is the maximum value of the rms relative fluctuation for a perfectly segregated system $(0,1$ bimodal (delta) distribution). Thus, $\mathbf{F}$ is the relative fluctuation for the system in the absence of molecular diffusion, and $X$ is the unmixedness relative to this maximum value. Normalization allows comparison of systems of different mean concentrations, $\bar{C}$, and bounds $X$ at 0 and 1 . Thus $\mathbf{X}=0$ corresponds to a perfectly mixed system, and $X=1$ a perfectly segregated system. It is noted that $X$ is a symetric quantity in that $\mathbf{X}_{\mathbf{j}}=\mathbf{X}_{\mathrm{m}}$. It was found that the mean value $\bar{C}$ determined from the combined optical and gas sampling measurements was approximately equal to the the metered value, therefore the metered value was used to compute F.

Because of the large number of pixels per frame, $X$ could be obtained with sufficient accuracy from a single $10 \mu \mathrm{s}$ exposure using the low light level vidicon camera. Several exposures were used, nevertheless, to compute $\mathbf{X}$ from the time-resolved data. Also, for this study, it was felt that determination of the mean mass fraction distribution was equally important. Thus, ensemble averaging of the entire field was determined from a 5-10 second time exposure. Also,

$$
\begin{aligned}
& \frac{C_{r m s}}{C_{a v g}}=\frac{\sqrt{\frac{1}{m}} \sum_{i=1}^{m}\left(\bar{C}_{i}-C_{a v g}\right)^{2}}{C_{a v g}} \\
& \text { where, } \\
& \quad C_{\text {avg }}=\frac{1}{m} \sum_{i=1}^{m} C_{i}
\end{aligned}
$$

In Eq. 5, Cij is replaced by the ensemble average at a point over all $n$ images, $\bar{C}_{j}$. It was found that $\mathbf{X}$, based on the unmixedness of the mean mass fraction distribution (Eq. 5 above), correlated well with $\mathbf{X}$ as determined from time-resolved measurements (Eq. 1). Unmixedness determined from time-resolved and time-averaged data are compared in Table 3. This indicates that the unmixedness based on 
the mean spatial distribution (Eq. 5) is less than that of Eq. 1, as would be expected. Spatial unmixedness (Eq. 5) is thus a useful measure of the total unmixedness.

\begin{tabular}{|c||c||c||c||c||c|}
\hline$J$ & $X / D=$ & 0.6 & 1.0 & 1.4 & 1.8 \\
\hline \multirow{3}{*}{5} & Eq 1 & 0.67 & 0.43 & 0.45 & 0.39 \\
& Eq 5 & 0.52 & 0.36 & 0.29 & 0.21 \\
\hline \multirow{3}{*}{18} & Eq 1 & 0.42 & 0.34 & 0.22 & 0.20 \\
& Eq 5 & 0.35 & 0.25 & 0.16 & 0.14 \\
\hline \multirow{2}{*}{78} & Eq 1 & 0.43 & 0.37 & 0.40 & 0.29 \\
& Eq 5 & 0.40 & 0.33 & 0.22 & 0.20 \\
\hline
\end{tabular}

Table 3: Comparison of Unmixedness for Timeresolved (Eq. 1) and Time-Averaged (Eq. 5) data

\section{Results and Discussion}

Experiments were conducted in two duct configurations, a wasp-waist configuration and a straight cylinder. The wasp-waist configuration was studied initially in support of ongoing combustion experiments in a can-type configuration, and the approach was to gather critical information as quickly as possible. The first series of tests was predicated on the assumption that the effect of orifice size should be relatively weak as observed in rectangular duct configurations ${ }^{1-5}$, and that, for a given momentum flux ratio, jet penetration and mixing should depend primarily on orifice spacing (i.e. the number of orifices). It was determined subsequently that unmixedness was affected by orifice size. Unmixedness also appears to be more sensitive to circumferential uniformity and less sensitive to jet penetration in a can than found previously in a rectangular duct. Further tests of a parametric nature appear warranted.

\section{Experiments in a Wasp-Waist Test Section}

The first series of experiments was done in the wasp-waist configuration with the 12 slot injector system. To be consistent with other investigations, momentum flux ratio, $J$, defined as the ratio $\rho_{j} V_{j}^{2} / \rho_{m} V_{m}{ }^{2}$, is treated as a primary variable, although density ratio was not varied initially. Unmixedness was measured as a function of momentum flux ratio by varying the slot size while holding the injectant mass flow constant. Slot dimensions are given in Table 2. A discharge coefficient of 0.6 was assumed in computing $V_{j}$ from the mass flow average velocity. The mass flow ratio was 1.16. Unmixedness, in duct diameters, is shown as a function of distance in Fig. 3 at three momentum flux ratios. The closest allowable measuring plane was at $X / D=0.6$, a location just downstream of the flange connecting the straight and diverging sections. The data indicate that substantial mixing has occurred prior to this location. Since the mass flow ratio was about unity, the unmixedness did not differ significantly from the relative unmixedness $\mathbf{X}$. The data suggest the existence of an optimum $J$, but it will be shown subsequently that this maybe a diameter effect (Fig. 5).

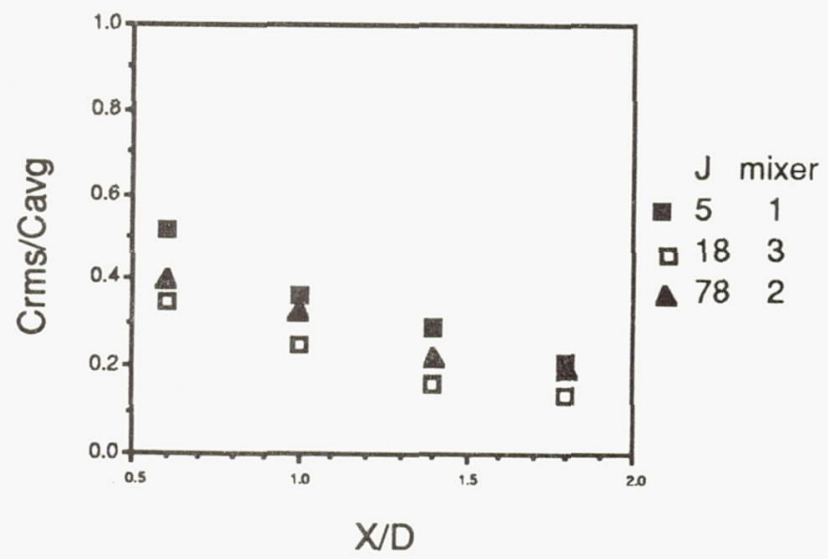

Figure 3: Effect of Momentum Flux Ratio on Unmixedness

(wasp-waist, 12 slots, $w_{j} / w_{m}=1.16$ )

In Fig. 4 mass fraction differences relative to the fully mixed condition are shown for the 6 slot injector at $X / D=0.6$. The middlelevel gray corresponds to the fully mixed condition. As $\mathrm{J}$ increases, the range of the concentration distribution decreases, indicating better mixing. 
Figure 4: Effect of Momentum Flux Ratio

$$
\begin{aligned}
& 6 \text { slanted slots } \\
& X / D=0.6
\end{aligned}
$$

Density Ratio $=1.0$
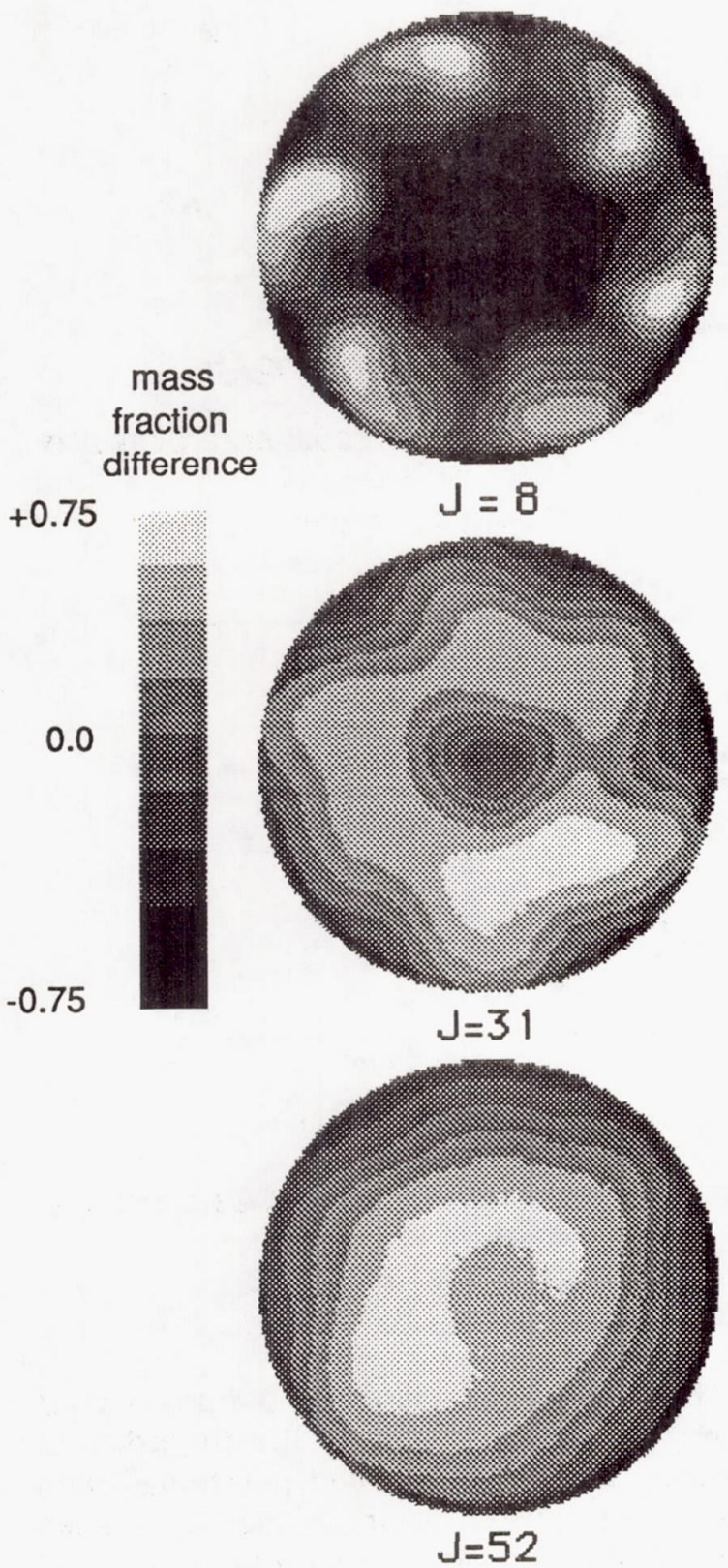

In order to explore the effect of orifice size a short series of experiments was run with size varying at constant momentum flux ratio. At $X / D=0.6$, where the overall level of unmixedness is still relatively high, Fig. 5, little difference is seen between configurations. Farther downstream at $X / D=1.0$, Fig. 6 , the data are segregated by orifice size (or mass flow ratio), for a given number of orifices. Mixing improves with increased mass flow.
Also shown in Fig. 6 are data from the 6 slot configuration at $X / D=1.0$ with the smallest slot. It is seen that, for the same orifice size, mixing is much more rapid in the 6 slot configuration.

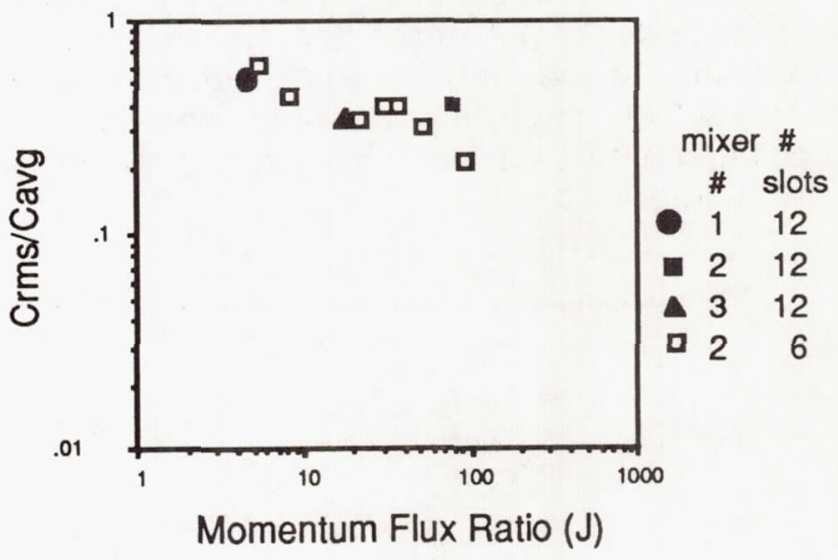

Figure 5: Effect of Orifice Size on Unmixedness at $X / D=0.6$

(wasp-waist)

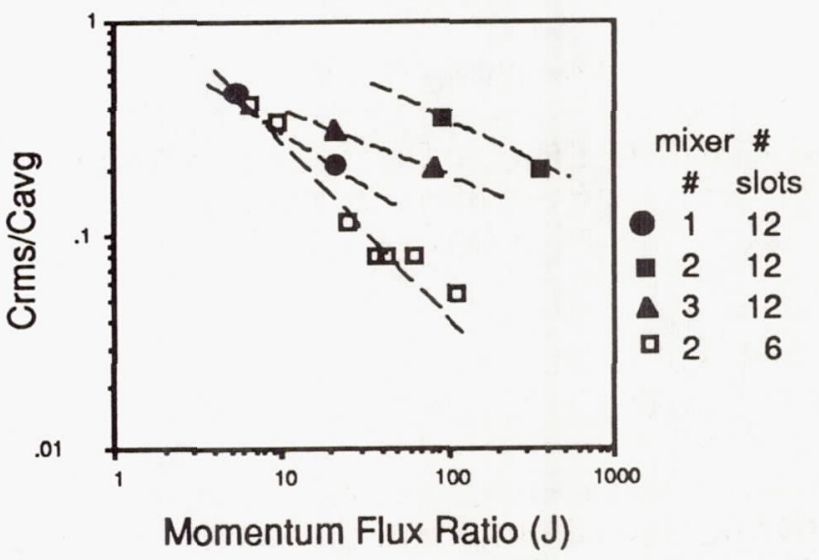

Figure 6: Effect of Orifice Size on Unmixedness at $X / D=1.0$

(wasp-waist)

It is noted that $\mathrm{J} \sim 30$ is a representative value for the HSCT combustor at the cruise condition, and the data from the 6 slot configuration suggest that low levels of unmixedness are attainable within one duct diameter. In the combustor application, a higher ratio of jet to mainstream mass flow is needed than in many of the tests in this study. The values here were chosen so that the resultant momentum flux ratios would be typical, independent of density ratio. Since, in the combustor application, the jet fluid would be more dense that that of the mainstream, the orifice areas studied here are typical. 
The relative unmixedness $x$, i.e. the unmixedness relative to its maximum theoretical value $F$, is shown in Fig. 7 at $X / D=1.0$ as a function of momentum flux ratio. With $\mathbf{X}$ as the dependent variable, the effect of orifice size is minimized for 12 slots, i.e. $X$ appears to be only a weak function of $\mathrm{J}$. Additional experiments with the 6 slot configuration and slot size varying would be very interesting.

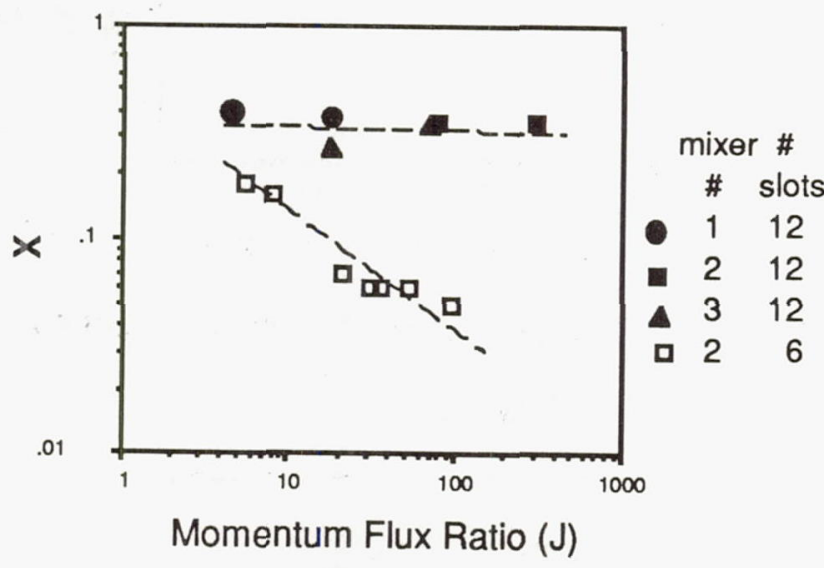

Figure 7: Effect of Orifice Size on Relative Unmixedness at $X / D=1.0$

(wasp-waist)

\section{Effect of Orifice Shape}

Unmixedness is compared in round hole and $45^{\circ}$ slot injector systems of equal area in Figs. 8 and 9 ( 6 orifices). At $X / D=0.6$ the two systems exhibit roughly the same behavior (Fig. 8). At $X / D=1.0$ (Fig. 9), however, it is evident that mixing with slot injectors is more rapid. The dependence of unmixedness on $\mathrm{J}$ is more complicated with round orifices.

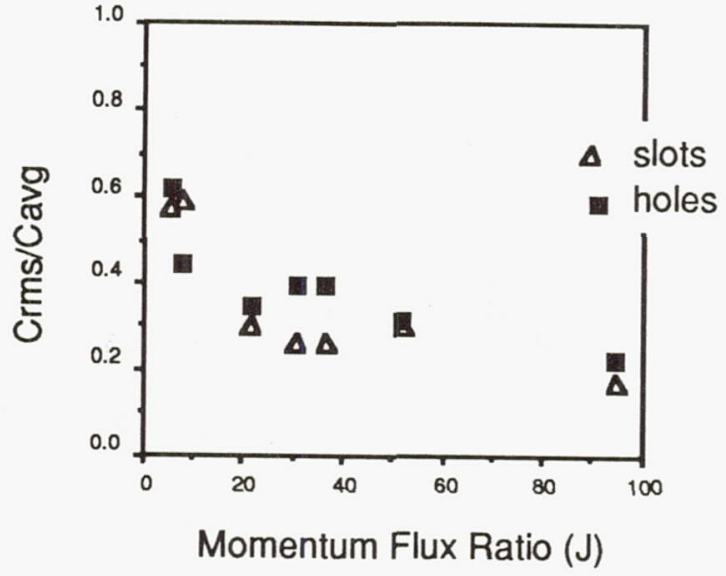

Figure 8: Unmixedness of Equal Area Slots and Holes at $X / D=0.6$

(wasp-waist, mixer 2)

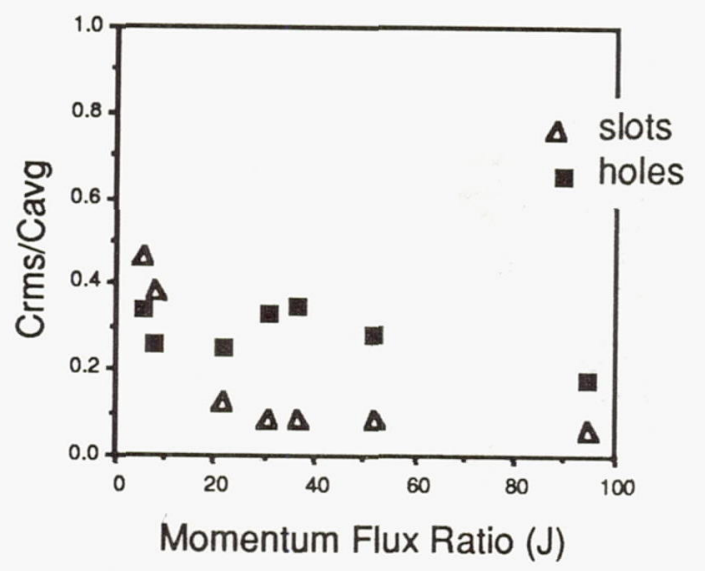

Figure 9: Unmixedness of Equal Area Slots and Holes at X/D $=1.0$

(wasp-waist, mixer 2)

Representative still frames from movies of low speed flows (Fig. 10a-d) indicate differences in the jet/jet and jet/mainstream dynamics of slots and round injectors. The jet exiting a round hole forms two counter-rotating vortices of equal strength. The jet penetrates directly toward the center of the duct and the jet cross section becomes more elongated as jet momentum increases. The connecting sheet moves closer to the duct axis, but the vortices tend to remain near the duct wall. 


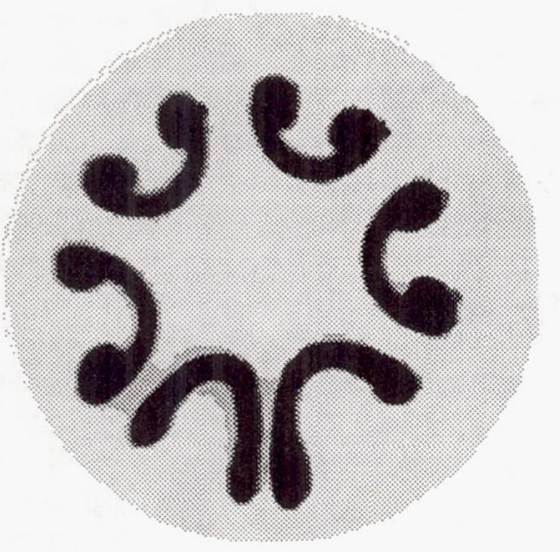

Figure 10a: Round Hole at low J

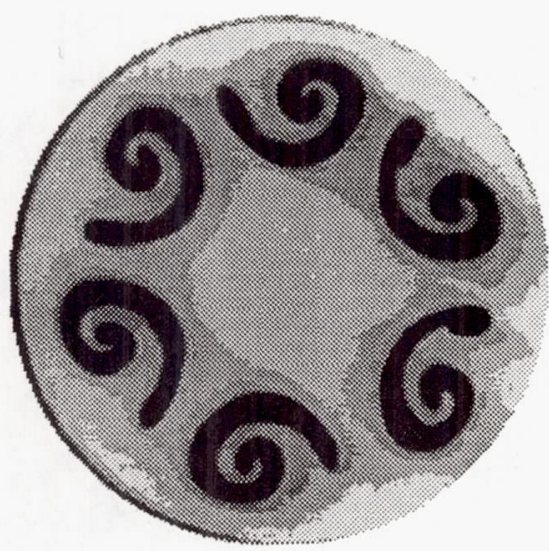

Figure 10c: Slanted Slot at low J

The tendency of the vortices to remain near the wall is attributed, in part, to the interaction between neighboring vortices from adjacent jets. Since neighbors rotate in an opposite sense, the induced velocity field is one that tends to translate neighbor pairs toward the duct wall. An additional influence of neighboring jets is to constrain the lateral spreading of the jet and stretch it along its midplane; this may contribute to the slightly greater observed penetration of round jets relative to elliptical slanted jets. In contrast to the round jet, the initially elliptical slanted jet forms a pair of counter-rotating vortices which are of unequal size and strength. The larger primary vortex forms at the leading edge of the slot and moves towards the duct wall while the smaller vortex moves away from the wall. There is considerable interaction between

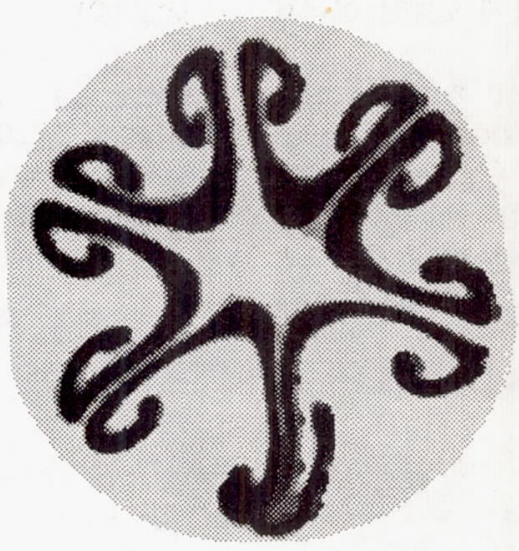

Figure 10b: Round Hole at higher $\mathrm{J}$

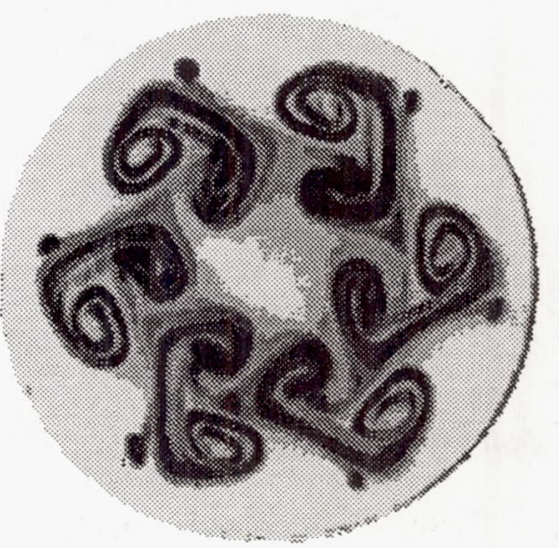

Figure 10d: Slanted Slot at higher $\mathrm{J}$

neighbors early in the injection process. In this case, unlike the round jet system, the induced velocity field is such that the vortex pair rotates about an axis connecting the vortex centers. At the same time, the smaller vortex undergoes a continual stretching deformation as it is drawn in by the upwash of its neighbor pair. It is speculated that this process may play a role in the overall mixing since the stretching process creates surface area while, at the same time, ingesting duct fluid. This would be in accord with the observation that mixing was found to be slightly more rapid with slanted slot injectors than with round injectors. Furthermore, the slanted jet experiences a side force which both tilts the jet and causes it to rotate about the duct axis. At the same time, the mainstream rotates in the opposite sense so that the net angular momentum of the system is zero. 


\section{Experiments in a Straight Mixing Section}

In order to minimize effects of duct geometry and better isolate the effects of flow and injector geometry, experiments were conducted in a straight cylinder. Momentum flux ratio, injector spacing, and density ratio were the primary variables.

\section{Effect of Duct Geometry}

It was first established that the unmixedness in the two duct configurations did not differ greatly. Unmixedness is compared in Fig. 11 for the two configurations at $X / D=0.6$ with 6 slot injection.

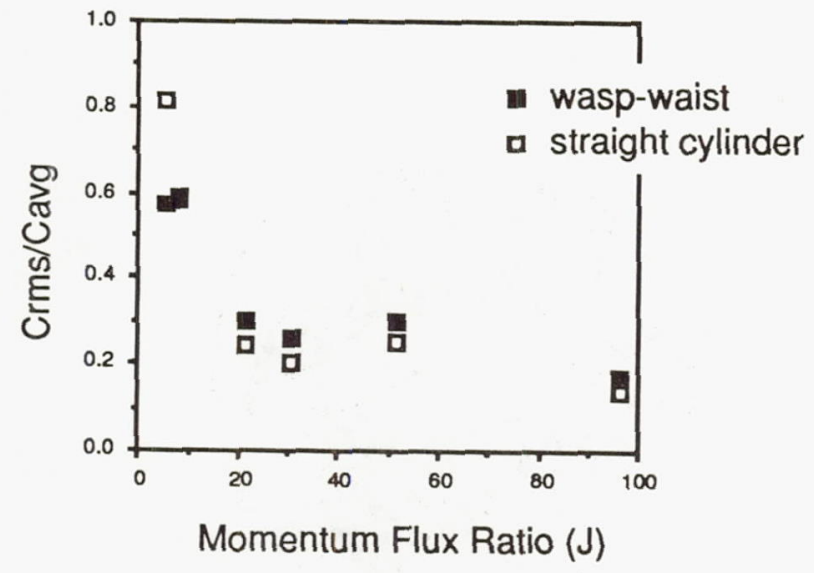

Figure 11: Effect of Duct Geometry on Unmixedness for 6 slot injection at $X / D=0.6$ (mixer 2)

To clarify the presentation the following comparisons are for the straight duct only.

\section{Effect of Density and Momentum Flux Ratio}

Figs. 12 and 13 show the dependence of unmixedness on momentum flux ratio for fixed orifice size (mixer 2) and spacing (6 slots) at two density ratios. The data indicate an optimum value of $\mathrm{J}$ (minimum unmixedness), but the effect diminishes with axial position. The optimum is attributed to over- and underpenetration of the jets at the extreme J's. It is seen that mixing is retarded at density ratio above unity. Therefore momentum flux ratio alone is not sufficient to characterize mixing. Again, at $X / D=1.0$ very low levels of unmixedness are achieved with the 6 slot injector.

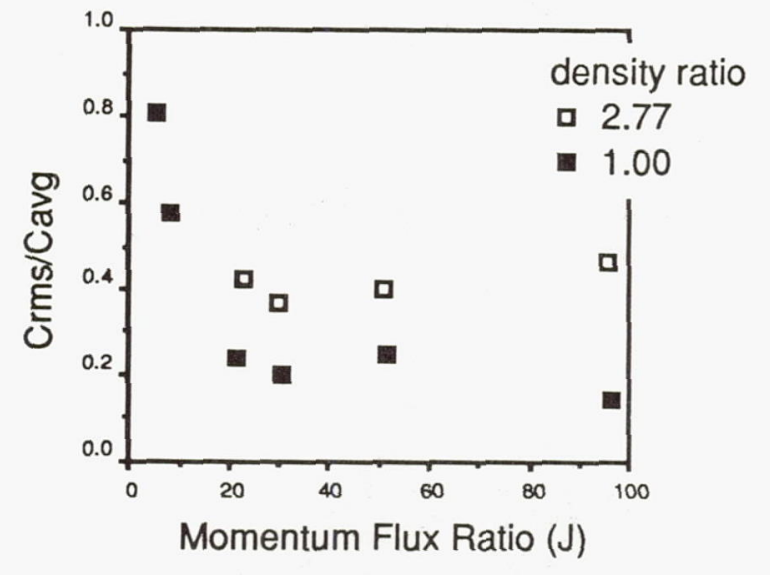

Figure 12: Effect of Momentum Flux Ratio on Unmixedness at $\mathrm{X} / \mathrm{D}=0.6$ (straight cylinder, mixer 2)

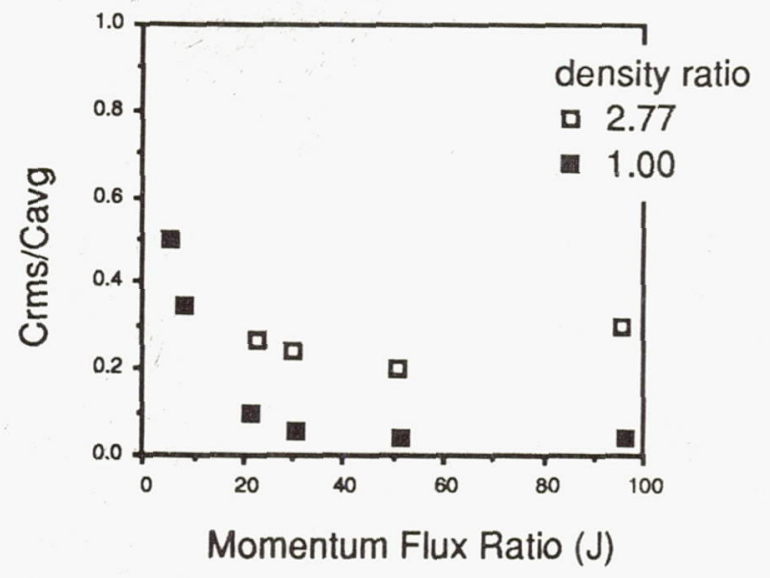

Figure 13: Effect of Momentum Flux Ratio on Unmixedness at $X / D=1.0$ (straight cylinder, mixer 2) 


\section{Effect of Orifice Spacing}

All of the data at density ratio unity are shown in Figs. 14 and 15 with orifice spacing as a parameter. It is evident that orifice spacing has a strong effect on mixing.

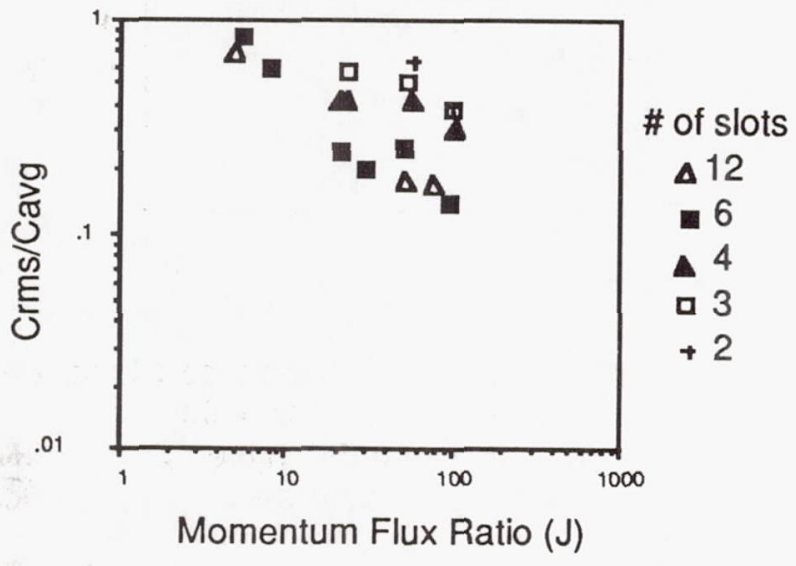

Figure 14: Unmixedness vs Momentum Flux Ratio at $X / D=0.6$ and Density Ratio $=1.0$ (straight cylinder, mixer 2)

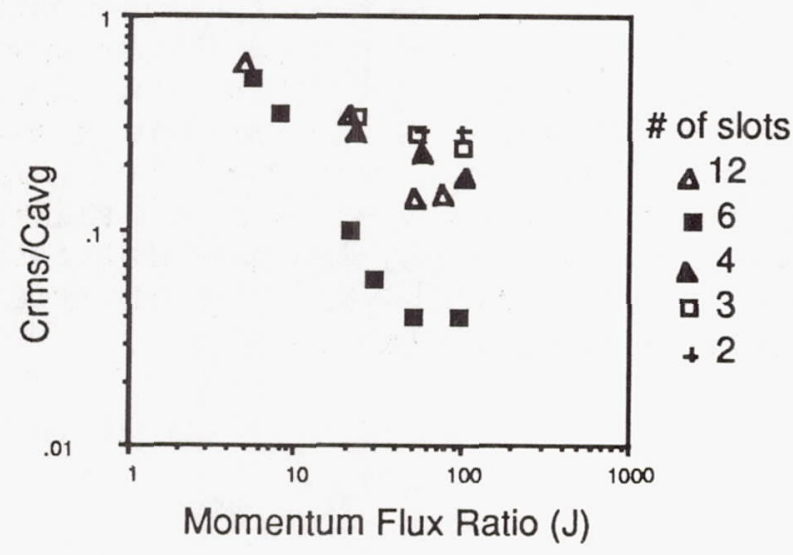

Figure 15: Unmixedness vs Momentum Flux Ratio at $X / D=1.0$ and Density Ratio $=1.0$ (straight cylinder, mixer 2)
Unmixedness is plotted as a function of slot spacing at two downstream positions in Fig. 16. The momentum flux ratio is 50 . The optimum number of slots is indicated to be between 6 and 12 .

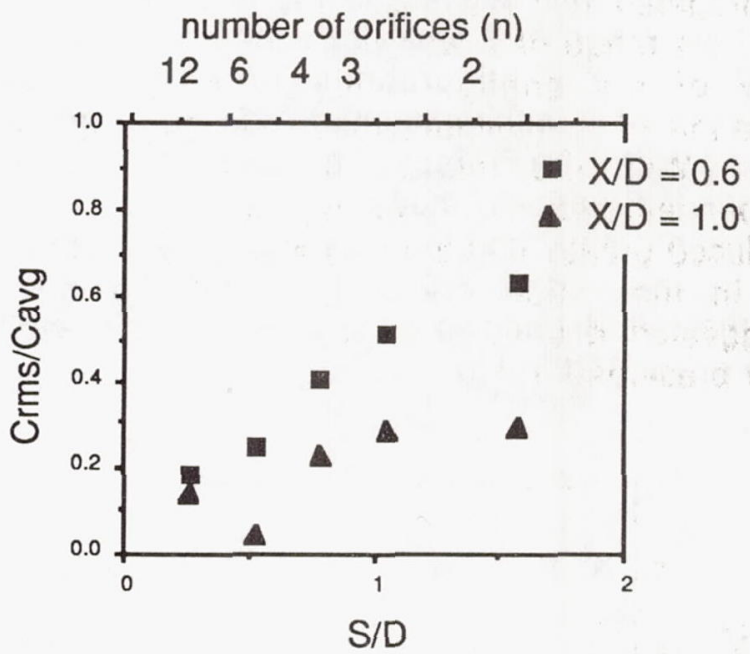

Figure 16: Effect of Hole Spacing on Unmixedness

(straight cylinder, mixer 2)

In an extended series of experiments in rectangular ducts, Holdeman, et al. 4,5 have shown, on the basis of inspection of mean temperature profiles, that for multi-source injection from equally spaced orifices in rows, jet penetration and mixing can be correlated with a single variable composed of the product of momentum flux ratio and hole spacing, independent of orifice size. The relationship between momentum flux ratio and hole spacing is given by the expression:

$$
\sqrt{ } \mathbf{J} * \mathbf{S} / \mathrm{H}=\mathbf{C}
$$

where,

$$
\begin{aligned}
& \mathrm{S}=\text { spacing } \\
& \mathrm{H}=\text { duct height } \\
& \mathrm{J}=\text { momentum flux ratio }
\end{aligned}
$$

optimum mixing was obtained with:

$\mathrm{C}=2.50$ for single side injection

$=1.25$ for 2 -sided directly opposed injection (inline)

$=5.00$ for 2 -sided staggered injection 
This relationship is tested for the present round configuration in Fig. 17 and 18 . Fig. 17 shows unmixedness as a function of the variable $\mathbf{Z}=\sqrt{ } \mathrm{J} * \mathrm{~S} / \mathrm{H}$ with orifice size held constant, mixer 2 . The number of slots varied from 2 to 12 . It is evident that the data are segregated with respect to the number of slots, and the range of $\mathbf{Z}$ was not sufficiently great for any of the configurations to establish the location of a minimum unmixedness (optimum $Z$ ). With the relative unmixednes as the dependent variable, however, the segregation is reduced greatly and the existence of an optimum $Z$ in the range $5.0>Z>2.5$ (for $X$ ) is suggested, depending on spacing. These results are presented in Fig. 18.

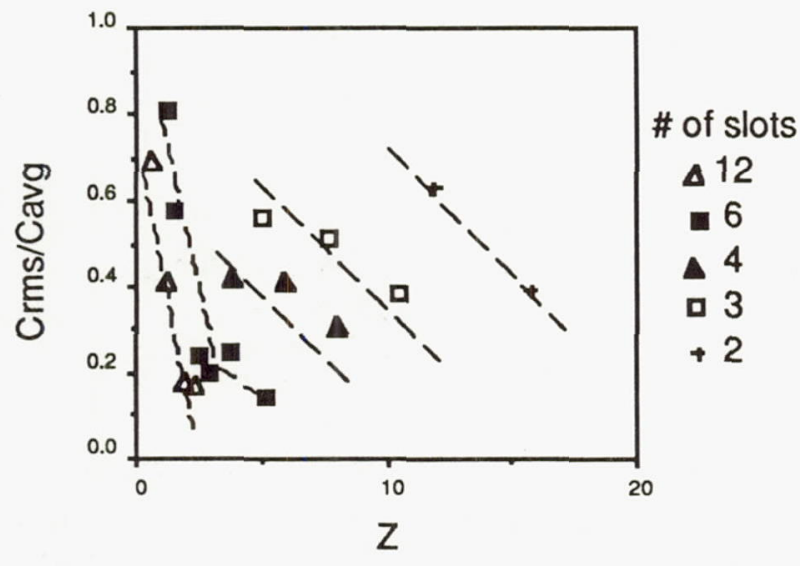

Figure 17: Unmixedness as a function of $\mathbf{Z}$ at $\mathrm{X} / \mathrm{D}=0.6$

(slot size constant, mixer 2)

(straight cylinder)

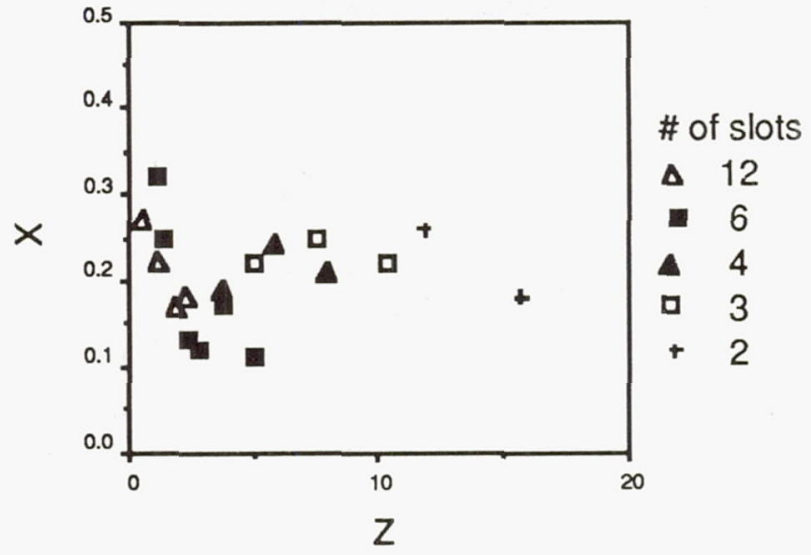

Figure 18: Relative Unmixedness $(\mathbf{X})$ as a function of $Z$ at $X / D=0.6$ (slot size constant, mixer 2) (straight cylinder)

The effects of pre-determined fluid mechanic and geometric variables have been described above for cross-stream mixing with multi-source injection. These studies were directed toward rapid screening of interesting configurations for application to mixing in an RQL combustor. The results indicate that acceptable levels of unmixedness are attainable in short distances at the relative flow rates needed. Extension of the variables is the subject of future work. More detailed analysis of the results is underway. Analytical studies which utilize the existing data base and relate mixing and $\mathrm{NO}_{x}$ formation are being done under a separate task of this contract. 


\section{Conclusions}

- At representative $\mathrm{J}$ and optimum spacing, low levels of unmixedness are attainable in one mixing passage duct height with slanted slot injectors.

- Mixing rate decreases with increasing density ratio.

- Relative unmixedness is nearly independent of orifice size and, hence, mass flow rate.

- Above a certain momentum flux ratio, mixing is faster with slanted slot injectors than with round hole injectors.

- Improved mixing with slanted slot injectors may be due to an initial unsymmetrical vortex pattern characteristic of slanted slots.

\section{Acknowledgements}

This work was funded under NASA Contract NAS3-25967.

\section{References}

1. Holdeman, J.D., "Mixing of Multiple Jets with a Confined Subsonic Crossflow; Summary of NASA Supported Experiments and Modeling," AIAA Paper 91-2458 (NASA TM 104412), June 1991.

2. Holdeman, J.D., Walker, R.E., and Kors, D.L., "Mixing of Multiple Dilution Jets with a Hot Primary Airstream for Gas Turbine Combustors," AIAA paper 731249 (NASA TM X-71426), Nov. 1973.

3. Holdeman, J.D. and Walker, R.E., "Mixing of a Row of Jets with a Confined Crossflow," AIAA Journal, Vol. 15, No. 2, Feb. 1977 (see also AIAA paper 76-48 (NASA TM-71821).
4. Holdeman, J.D., Srinivasan, R., and Berenfeld, A., "Experiments in Dilution Jet Mixing," AIAA Journal, Vol. 22, No. 10, Oct. 1984 (see also AIAA Paper 831201 (NASA TM-83434)).

5. Holdeman, J.D., Srinivasan, R., Coleman, E.B., Meyers, G.D., and White, C.D., "Effects of Multiple Rows and NonCircular Orifices on Dilution Jet Mixing, J. of Propulsion and Power, Vol. 3, No. 3, May-Jun 1987 (see also AIAA paper 851104 (NASA TM 86996)).

6. Smith, C.E., Talpallikar, M.V., and Lai, M.C., "Rapid Mix Concepts for Low Emission Combustors in Gas Turbine Engines," NASA CR-185292, October 1990, pp. 2.

7. Smith, C.E., Talpallikar, M.V., and Holdeman, J.D., "A CFD Study of Jet Mixing in Reduced Flow Areas for Lower Combustor Emissions,"

AIAA Paper 91-2460 (NASA TM 104411), June 1991.

8. Vranos, A. and Liscinsky, D.S., "Planar Imaging of Jet Mixing in Crossflow," AIAA Journal, 26, 11, November 1988, pp 1297-98. 


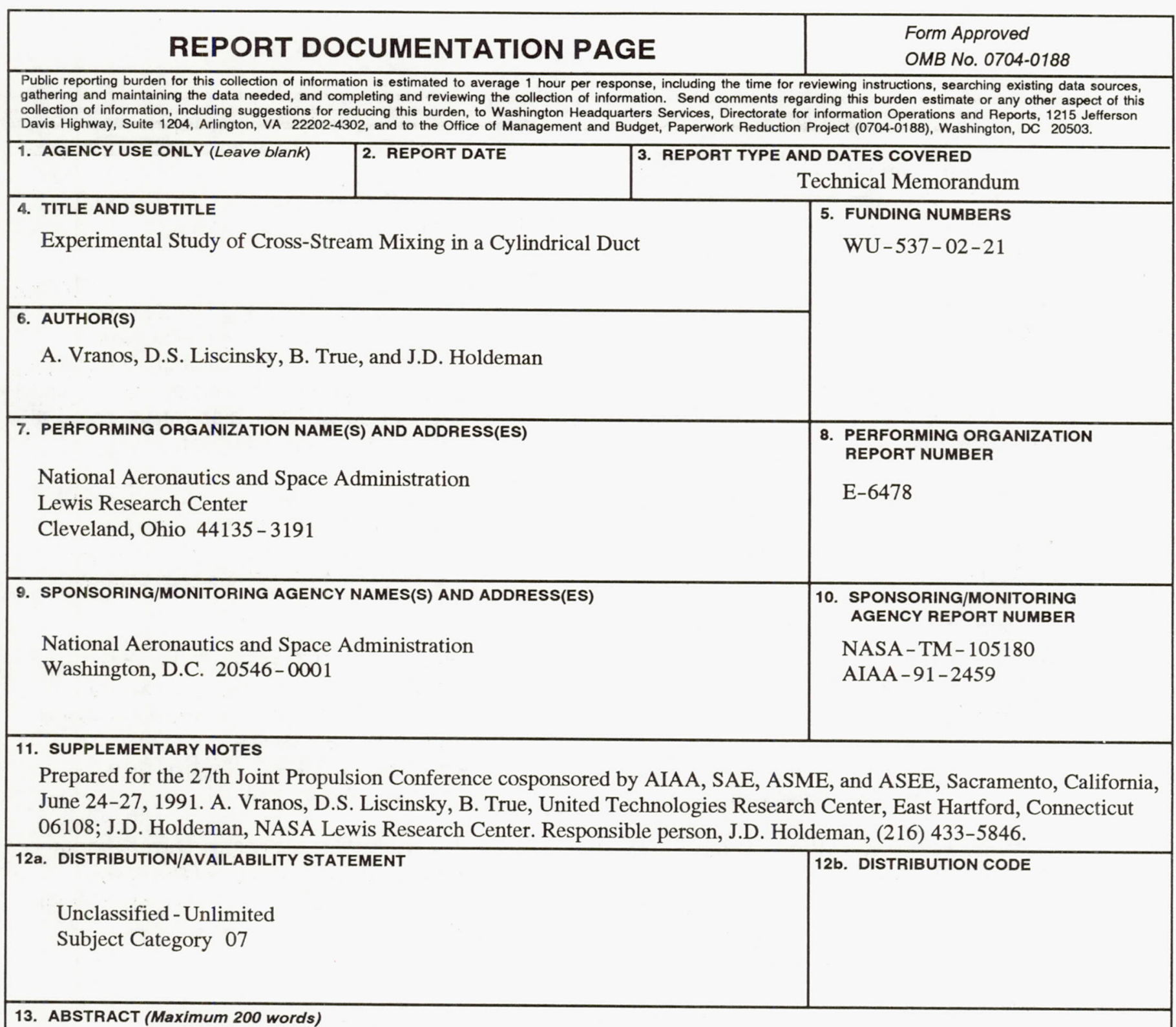

An experimental investigation of cross stream injection and mixing has been conducted with application to a low $\mathrm{NO}_{\mathrm{x}}$ combustor for the HSCT. Mixing in a cylindrical chamber has been studied for transverse injection from slanted slot and round orifice injectors. Momentum ratio, density ratio, and injector geometry were the primary variables. Slanted slots of various size, aspect ratio, and number were studied. Quantitative measurement of injectant concentration distributions were obtained by planar digital imaging of the Mie-scattered light from an aerosol seed uniformly mixed with the injectant. The unmixedness, defined as the ratio of the r.m.s. concentration fluctuation to mean concentration in a plane perpendicular to the main flow direction, was found to be primarily a function of momentum ratio and injector spacing. An optimum spacing is indicated. Unmixedness is also a function of orifice size, or mass flow ratio, but the mass flow dependence can be accounted for by normalizing the unmixedness with its maximum theoretical value. The data indicate that a density ratio greater than unity retards mixing. It was found that above a certain momentum flux ratio, mixing with slanted slot injectors was better than with round hole injectors. Video tapes of the mixing at very low stream velocity indicate different jet/jet and jet/mainstream interactions for the two types of injectors.

\section{SUBJECT TERMS}

Dilution; Jet mixing flow; Gas turbines; Combustion chamber; Can; Emissions

15. NUMBER OF PAGES 14

16. PRICE CODE $\mathrm{A03}$

\begin{tabular}{|c|c|}
\hline $\begin{array}{c}\text { 17. SECURITY CLASSIFICATION } \\
\text { OF REPORT }\end{array}$ & $\begin{array}{c}\text { 18. SECURITY CLASSIFICATION } \\
\text { OF THIS PAGE } \\
\text { OFsified }\end{array}$ \\
\begin{tabular}{c} 
Unclassified \\
\hline
\end{tabular}
\end{tabular}
19. SECURITY CLASSIFICATION OF ABSTRACT Unclassified

20. LIMITATION OF ABSTRACT 